\title{
Miniaturized Infrared Spectrometer based on Planar Focusing Grating
}

\author{
Torsten May ${ }^{1}$, Andreas Wuttig ${ }^{2}$, Anja Wagner ${ }^{3}$, Alexander Brown ${ }^{1}$, Ernst Keßler ${ }^{1}$, Uwe Hübner ${ }^{1}$, Elmar \\ Voigt $^{1}$, Rainer Riesenberg ${ }^{1}$, Hans-Georg Meyer ${ }^{1}$ \\ ${ }^{1}$ Leibniz-Institut für Photonische Technologien, Albert-Einstein-Str. 9, 07745 Jena, Germany \\ ${ }^{2}$ Codion Optics GmbH, Wildenbruchstr. 15, 07745 Jena, Germany \\ ${ }^{3}$ Analytik Jena AG, Konrad-Zuse-Straße 1, 07745 Jena
}

\begin{abstract}
Planar gratings for applications in infrared spectrometers have been prepared with curved, nonequidistant groves by lithography and a deep etching process. The gratings feature focusing properties as well as the ability to tailor the spectral dispersion dependency, allowing a compact dispersive IR spectrometer. We report on the design, fabrication and characterization measurements. Based on these results, a concept for a compact spectrometer prototype utilizing thermoelectric detector arrays with high detectivity was developed.
\end{abstract}

Key words: infrared grating, spectrometer, thermoelectric detector, linear array, DRIE.

\section{Introduction}

Infrared spectroscopy has a broad range of applications since it can be used to detect molecules based on their characteristic vibrations. Application fields for such an analysis range from gas diagnostics to the observation of life processes. In the case of large biomolecules, the long-wave part of the infrared spectrum is particularly revealing. Measurement objectives in this area include food quality assurance [1] (detection of decomposition byproducts) or medical diagnosis [2] (e. g. in-vivo determination of blood sugar level).

In the long-wave infrared region, interferometrybased spectrometers (e. g., Fourier Transform spectrometers, FT-IR) are typically used for laboratory analysis. Such devices possess an unrivaled resolution but are not, due to their complex design, well-suited for on-site application. Spectrally analyzing light using an optical grating as a dispersive element is an established alternative with the prospect of a much simpler setup and a higher robustness, whilst still having a substantial advantage over non-dispersive IR spectroscopy (NDIR) in terms of selectivity.

Conventional grating spectrometers use classic gratings with straight, equidistant lines. Yet, irregular gratings with curved, non-equidistant lines have the potential to tailor the dispersion and to implement additional optical focusing, thus allowing for the design of miniaturized and inexpensive spectrometers for life-science and industrial applications.

\section{Spectrometer design}

Several different types of gratings being particularly promising for compact spectrometers have been analyzed by numerical simulation with the Zemax software. In view of the intended application in analytical methods, a flat wavenumber dispersion characteristic has been pursued because it is advantageously suited for chemometrics. In order to demonstrate the degrees of freedom, the simulation results of three different designs are shown in the following (Fig. 1-3).

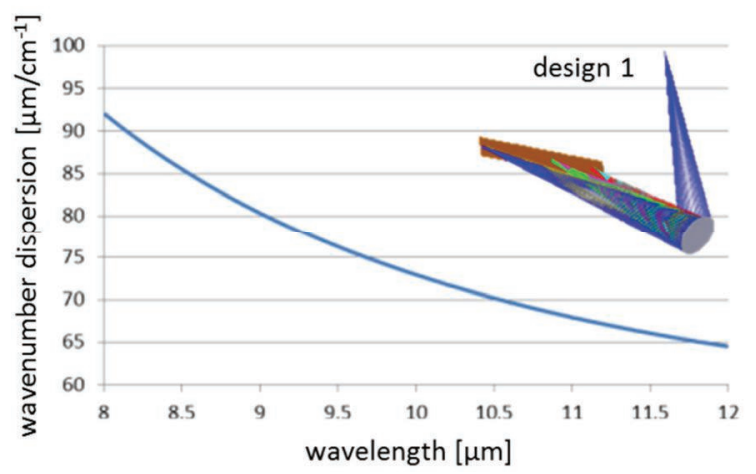

Fig. 1: Design 1: falling wavenumber dispersion with wavelength; geometrical dimension $66 \times 58 \times 10$ $\mathrm{mm}^{3}$ 


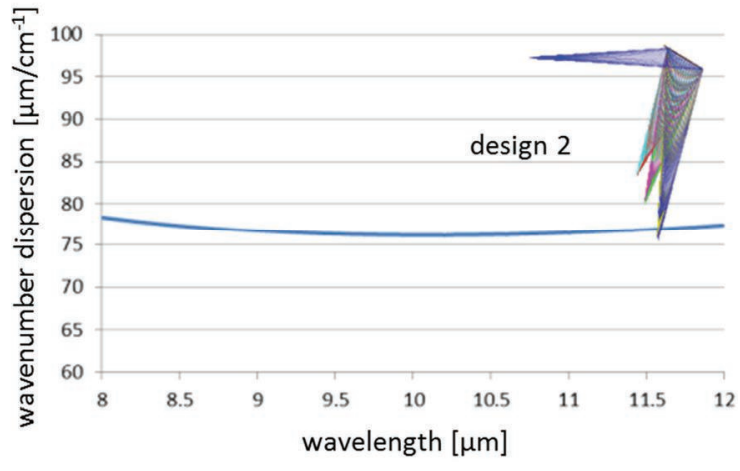

Fig. 2: Design 2: almost flat wavenumber dispersion with wavelength; geometrical dimension $91 \times 82 \times 10$ $\mathrm{mm}^{3}$

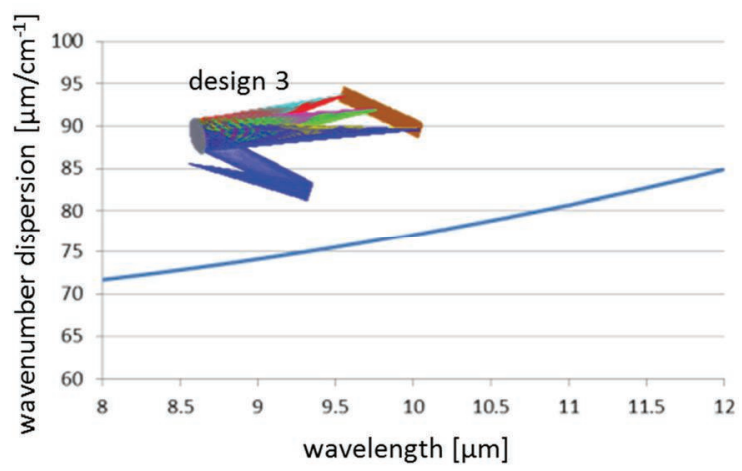

Fig. 3: Design 3: rising wavenumber dispersion with wavelength; geometrical dimension $90 \times 57 \times 10$ $\mathrm{mm}^{3}$

All three designs have the advantage of requiring max. one focusing mirror (design 1 and 2 require none at all), easing the adjustment and making a derived spectrometer more robust. In view of the intended miniaturization, another, even more compact design was chosen at the expense of the requirement of two focusing mirrors (see Fig. $4)$. Despite the higher complexity, this design has the main advantage of the required construction volume being almost a factor of two lower compared to designs 1, 2 and 3.

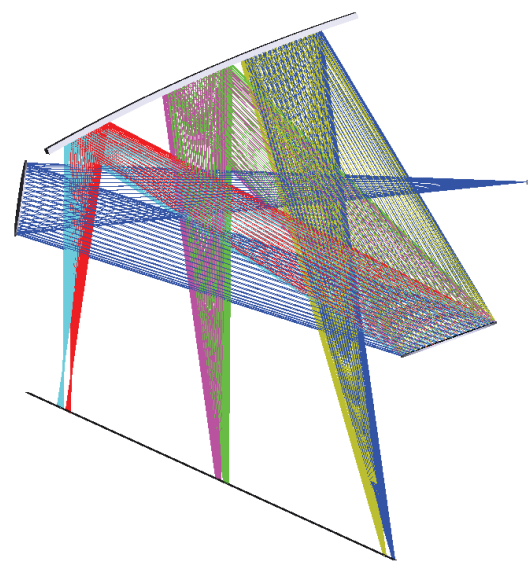

Fig. 4: Design 4: irregular grating and two focusing mirrors; geometrical dimension $44 \times 43 \times 7 \mathrm{~mm}^{3}$
Design 4 was selected to be the basis for the subsequent technical realization and characterization. The main parameters of design 4 are summarized in Tab. 1 .

Tab. 1: Parameters of design 4 (see Fig. 4)

\begin{tabular}{|c|c|}
\hline parameter & value \\
\hline length of image plane & $32 \mathrm{~mm}$ \\
\hline magnification & $1.01: 1$ \\
\hline linear dispersion & $7,6-8,8 \mu \mathrm{m} / \mathrm{nm}$ \\
\hline wavelength range & $8-12 \mu \mathrm{m}$ \\
\hline spectral resolution* & $85-100 \mathrm{~nm}$ \\
\hline
\end{tabular}

* spectral resolution defined by 3-Pixel-criterion using a 64 pixel detector array with $32 \mathrm{~mm}$ length; corresponding to IPHT type ZS64

\section{Grating technology}

The required grating line width and depth in the order of a few micrometers represent a challenge for conventional preparation techniques; especially if an edge roughness in the sub-100 $\mathrm{nm}$ range is required for a high image quality and a low scattering background. Therefore, a deep etching process in silicon was used that exhibits significantly higher etching rates than commonly used glass substrates and thus allows for an improved structural control.

Manufacturing was based on the so called cryo process [3], where a silicon single crystal substrate is etched by means of reactive ion etching (RIE). During the process, inductively coupled plasma (ICP) of a $\left(\mathrm{SF}_{6}+\mathrm{O}_{2}+\mathrm{CHF}_{3}\right)$ gas mixture enables sidewall passivation by $\left(\mathrm{SiO}_{x} \mathrm{~F}_{\mathrm{y}}\right)$. In comparison to the commonly used cyclical Bosch process, the continuous cryo process allows for smoother patterns. The process was established on a Sentech SI-500 tool at ICP power of $500 \mathrm{~W}$ and a process temperature of $-130^{\circ} \mathrm{C}$.

To create the grating patterns with a typical etch depth of about 5 to $10 \mu \mathrm{m}$, a hard mask from $\mathrm{SiO}_{2}$ was used which achieves the required stability against the cryo process. That $\mathrm{SiO}_{2}$ mask was patterned by means of electronbeam lithography which has the advantage of a fast transfer of new designs to technology development. However, in case of completed design development, series production will be accomplished by optical lithography using a wafer stepper, which promises a huge speed advantage over the time-consuming direct ebeam transfer. 


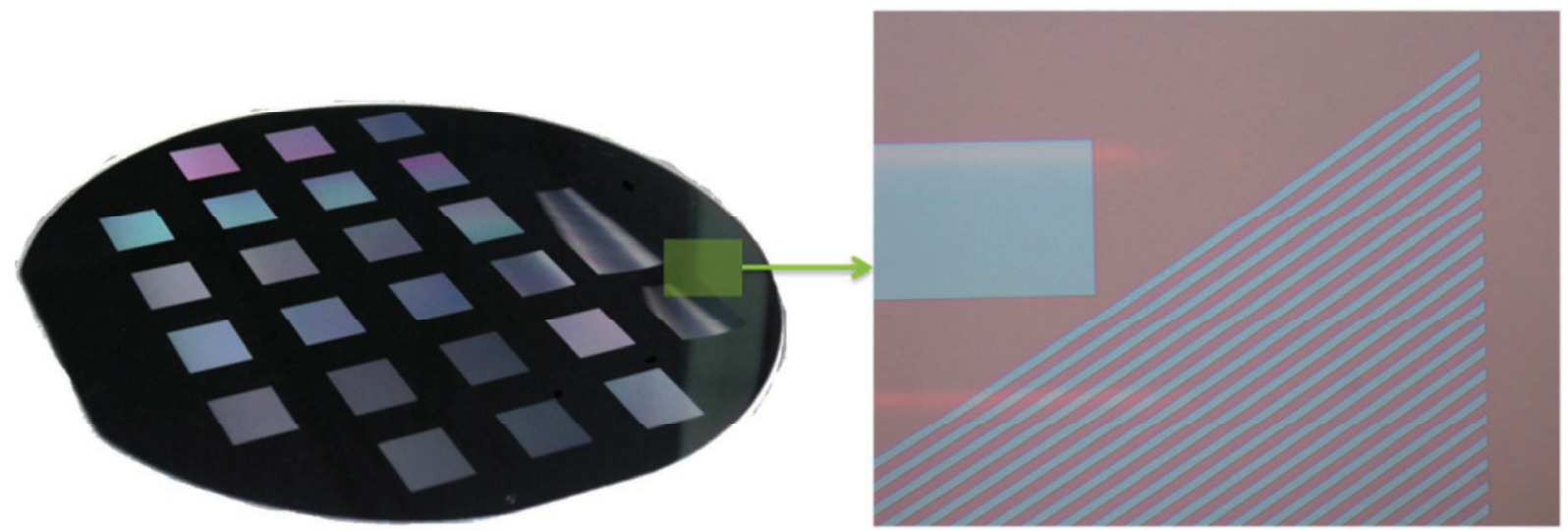

Fig. 5: 4" silicon wafer with different designs (left) and optical microscope image of a grating structure

In developing the appropriate technology, a strong dependence of the etch rate on the actual grating geometry (filling factor) was observed. For the described design 4, a depth of $3.9 \mu \mathrm{m}$ was required, which was achieved at a gas pressure of $0.6 \mathrm{~Pa}$. The etching time was altogether $18 \mathrm{~min}$. The resulting pattern is displayed in Fig. 6.

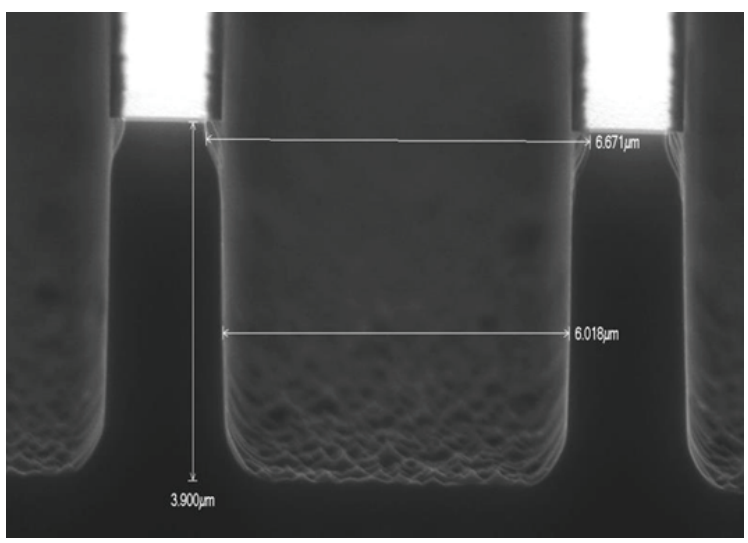

Fig. 6: Scanning electron microscope images of the grating pattern of design 4

The final manufacturing step is the deposition of $100 \mathrm{~nm}$ gold by electron beam evaporation. In order to achieve a homogeneous covering of the grating pattern (including the sidewalls), the deposition was performed at an angle of $45^{\circ}$ onto a rotating substrate.

\section{Experimental results}

In order to experimentally determine the performance of the micro-fabricated gratings, an evaluation model (Fig. 7) was assembled and measured using a thermal infrared line source $(\lambda=10 \mu \mathrm{m})$ and a laser light source $(\lambda=661 \mathrm{~nm})$. The latter much more clearly exposes imperfections in the grating.

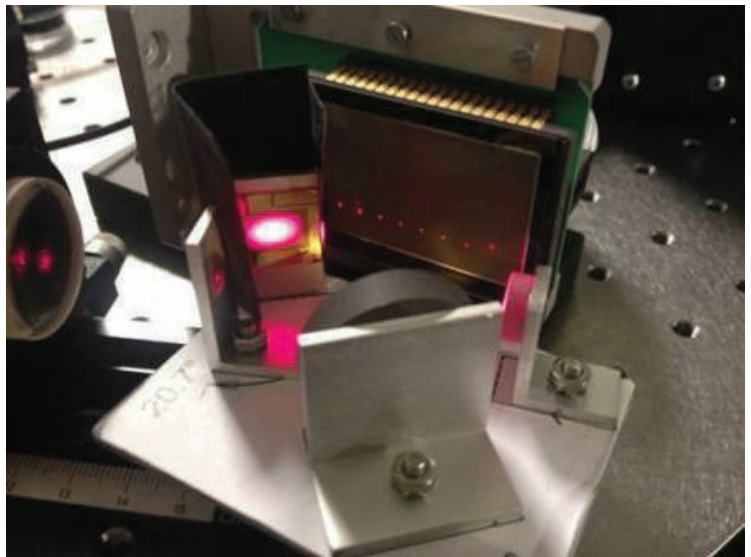

Fig. 7: Laboratory test setup of the spectrometer design 4 for stray light measurements; including a Laser source at $\lambda=661 \mathrm{~nm}$

It has been shown that the measured resolution corresponds to the simulation, whilst the diffraction efficiency reaches about $80 \%$ of the efficiency of an ideal binary grating. The total integrated scatter measured using the laser source at the diffraction plane was $<1.5 \%$ (contrast ratio approx. 2500:1), which yields practically negligible scattering in the target spectral range.

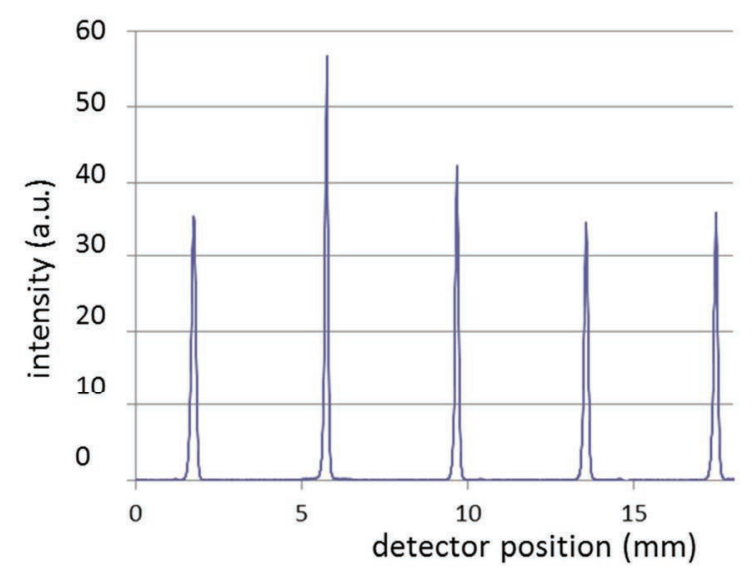

Fig. 8: Straylight measurement with a Laser at $\lambda=661 \mathrm{~nm}\left(12^{\text {th }}\right.$ to $16^{\text {th }}$ diffraction order $)$ 


\section{Theoretical performance}

Based on the promising results of the grating characterization, an operational spectrometer was developed. For that, a new optical design was developed, which bases on design 4 but uses another array sensor (see below). Moreover, further optimization of the design was performed based on previous experience.

First, a detailed set of achievable parameters has been calculated. The spectral range of the spectrometer was chosen to be between $1500 \mathrm{~cm}^{-1}$ and $2000 \mathrm{~cm}^{-1}$. As infrared source, a thermal thin-film source (model JSIR350-4-ALC-D5.8 from Microhybrid, Germany) at $\mathrm{T}=640^{\circ} \mathrm{C}$ is used. The dispersed spectrum is measured with a linear array ZS2×128 (having two staggered sub-arrays of 128 pixels each; see Fig. 9) which was manufactured at IPHT. The thermoelectric sensors are based on the thermocouple bismuth and bismuth/antimony which enables a very high detectivity $D^{*}$ in the order of $10^{9} \mathrm{~cm} \cdot \sqrt{\mathrm{Hz}} / \mathrm{W}$ [4]. By using an almost perfect silver soot absorber, an absorption $>99.5 \%$ across the entire relevant spectral range is achieved. Another advantage for the use in a spectrometer is the high linearity over about 5 orders of magnitude of the thermoelectric converter principle in which, according to the Seebeck effect, a temperature gradient generates an active voltage in the thermo element.

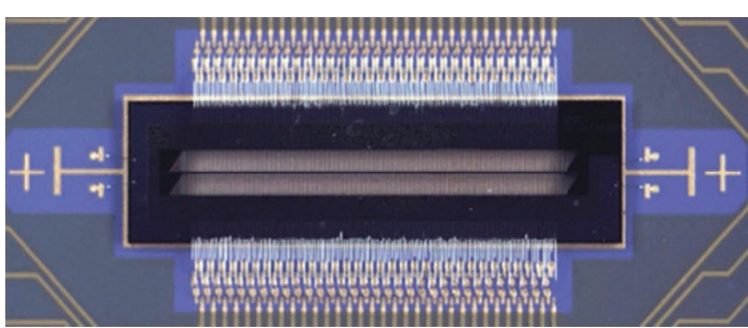

Fig. 9: Linear thermoelectric array ZS2×128

In order to calculate the spectrometer performance, the following data set of the detector array is relevant (Tab. 2).

Tab. 2: Main parameters of the linear thermoelectric array ZS2×128

\begin{tabular}{|c|c|}
\hline parameter & value \\
\hline pixel size & $0.089 \times 0.6 \mathrm{~mm}^{2}$ \\
\hline pixel pitch & $0.088 \mathrm{~mm}^{*}$ \\
\hline detectivity $\mathrm{D}^{*}$ & $0.9 \cdot 10^{9} \mathrm{~cm} \mathrm{~Hz}^{1 / 2} / \mathrm{W}$ \\
\hline response & $400 \mathrm{~V} / \mathrm{W}$ \\
\hline time constant & $100 \mathrm{~ms}$ \\
\hline resistance & $15 \mathrm{k} \Omega$ \\
\hline
\end{tabular}

* pitch is measured between two staggered pixels; one pixel in the upper sub-array and the other in the lower sub-array

The spectral emission $E(\lambda, A)$ of the thin film source mentioned above at $\mathrm{T}=640^{\circ} \mathrm{C}$ varies from $4.6 \cdot 10^{9} \mathrm{~W} \cdot \mathrm{m}^{-2} \cdot \mathrm{m}^{-1}$ at $2000 \mathrm{~cm}^{-1}(\lambda=5 \mu \mathrm{m})$ to $2.6 \cdot 10^{9} \mathrm{~W} \cdot \mathrm{m}^{-2} \cdot \mathrm{m}^{-1}$ at $1500 \mathrm{~cm}^{-1}(\lambda=6,7 \mu \mathrm{m})$. Assuming an entrance slit corresponding to the pixel dimensions (width approx. $100 \mu \mathrm{m}$, height $600 \mu \mathrm{m})$, the radiating area of $A=6 \cdot 10^{-8} \mathrm{~m}^{2}$ translates to a total power $\mathrm{P}=0.36 \mathrm{~mW}$ in the chosen spectral range. The optical design 4 allows for a throughput of about $2 \%$, thus, the power on the grating is $12,8 \mu \mathrm{W}$. As a rough assumption of a linear dispersion of the available power on 256 pixels at a grating efficiency of $50 \%$, the medium radiation power on a single pixel would be about $25 \mathrm{nW}$. In reality, the pixel at the shortest wavelength detects approx. $20 \%$ higher power, whereas the pixel at longest wavelength is illuminated with $20 \%$ less power.

At a response of $400 \mathrm{~V} / \mathrm{W}$, the maximum achievable signal is about $10 \mu \mathrm{V}$. Assuming a modulation of the optical source at $1 \mathrm{~Hz}$ in order to eliminate drifts, an electrical bandwidth of $2 \mathrm{~Hz}$ is required for the detector readout. In good approximation, the noise of the thermoelectric sensor is dominated by Johnson noise of its electrical resistance $(15 \mathrm{k} \Omega)$, which translates to a voltage noise of $15 \mathrm{nV} / \sqrt{\mathrm{Hz}}$ or, at a bandwidth of $2 \mathrm{~Hz}$, to $\mathrm{V}_{\mathrm{RMS}}=21 \mathrm{nV}$. In consequence, a spectrum between 100\% absorption $\left(\mathrm{V}_{\text {signal }}=0 \mathrm{~V}\right)$ and $0 \%$ absorption $\left(\mathrm{V}_{\text {signal }}=10 \mu \mathrm{V}\right)$ could be resolved at approx. 8 bit resolution $(10 \mu \mathrm{V} / 21 \mathrm{nV}=470)$. An accordingly higher signal-to-noise ratio can be achieved by integration over several subsequent spectra.

\section{Spectrometer design}

As a proof of concept, a compact table-top spectrometer was designed. The design bases on a vacuum chamber which encloses the detector array and the optical path in order to avoid interference due to absorption by atmospheric water vapor. A liquid sample can be placed in the path utilizing a standard cuvette for infrared measurements (Bruker liquid cell A140). The analogue electronics multiplexes 32 detectors per altogether 8 output channels which are digitized using standard 18 bit $A / D$ converters.

A layout of a complete spectrometer is displayed in Fig. 10. 


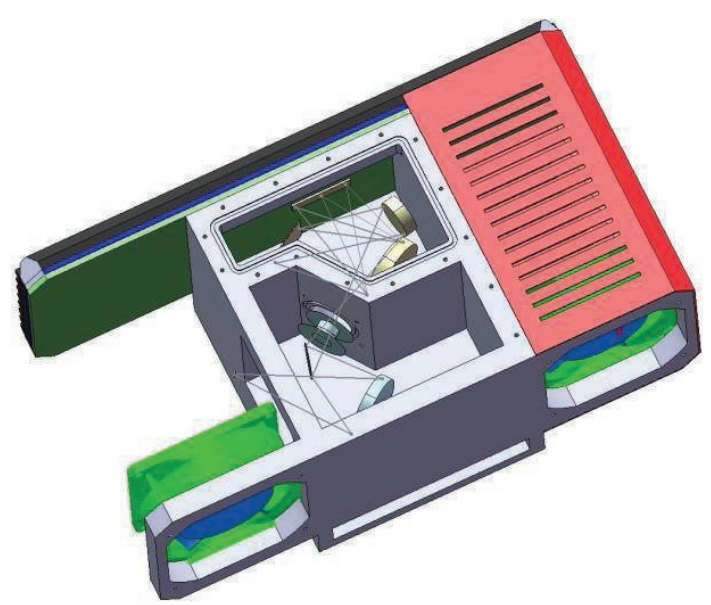

Fig. 10: $C A D$ model of a table-top spectrometer configuration

\section{Conclusion and outlook}

An infrared grating spectrometer was designed which features a spectral resolution of $\Delta \lambda \sim$ 20nm (3-Pixel criterion; optical FWHM $\sim 15 \mathrm{~nm}$ ) in the spectral range $1500-2000 \mathrm{~cm}^{-1}$. By employing a highly sensitive thermoelectric sensor line with 256 sensors, 8bit signal resolution could be achieved at $1 \mathrm{~Hz}$ integration time. The use of irregular gratings with focusing properties enables a very compact design, as demonstrated at a complete spectrometer layout of about $10 \times 20 \times 10 \mathrm{~cm}^{3}$, including electronics, cooling and sample feed.

\section{Acknowledgement}

Financial support by the Thüringer Aufbaubank (TAB) is acknowledged (project VITAL, grant No. 2011 FE 9081 / 2011 VF 0027)

\section{References}

[1] D. Cozzolinoa, Recent Trends on the Use of Infrared Spectroscopy to Trace and Authenticate Natural and Agricultural Food Products, Applied Spectroscopy Reviews 47(7), 518-530 (2012); doi:10.1080/05704928.2012.667858

[2] C. Hughes, M. Brown, G. Clemens, A. Henderson, G. Monjardez, N. W. Clarke, P. Gardner, Assessing the challenges of Fourier transform infrared spectroscopic analysis of blood serum, Journal of Biophotonics 7(3-4), 180-188 (2014); doi: 10.1002/jbio.201300167

[3] R. Dussart, T. Tillocher, P. Lefaucheux, M. Boufnichel, Plasma cryogenic etching of silicon: from the early days to today's advanced technologies, Journal of Physics D: Applied Physics 47(12), 123001 (2014); doi:10.1088/0022-3727/47/12/123001

[4] F. Haenschke, E. Kessler, U. Dillner, A. Ihring, U. Schinkel, H.G. Meyer, A new high detectivity room temperature linear thermopile array with a $D^{*}$ greater than $2 \times 109 \mathrm{cmHz} 1 / 2 / \mathrm{W}$ based on organic membranes, Microsystem technologies 19(12), 1927-1933 (2013); doi:10.1007/s00542013-1764-5 